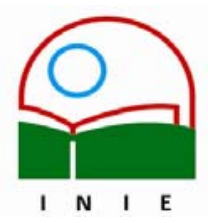

Universidad de Costa Rica

Facultad de Educación

Instituto de Investigación en Educación

ACTUALIDADES INVESTIGATIVAS EN EDUCACION

\title{
EL APORTE DE LA NEUROCIENCIA PARA LA FORMACIÓN DOCENTE
}

\begin{abstract}
Susan Francis Salazar ${ }^{1}$
Resumen: El siguiente artículo se propone, a partir del análisis de un proceso de investigación sobre el área pedagógica en los planes de formación docente, realizar una invitación a la incorporación del estudio del cerebro humano, como eje dentro de la formación profesional para el ejercicio docente. La capacidad de educabilidad propia de los seres humanos tiene su explicación sobre la base del aporte de distintas disciplinas científicas; sin embargo, en ocasiones hemos focalizado nuestra atención en aspectos psicológicos y filosóficos, sin considerar que lo biológico juega un papel trascendental. Algunas situaciones presentes en el acto cotidiano escolar encuentran su origen en la actividad neuronal humana. Por ello el conocimiento que el docente tenga sobre sus características, potencialidades $y$, por ende, sobre las implicaciones en los distintos ámbitos de la acción educativa le permite ampliar las fuentes para la toma de decisiones en los procesos pedagógicos. Pensar en el desarrollo profesional del docente, exige crear y promover espacios de construcción del conocimiento acerca de la manera como los seres humanos nos educamos y los elementos que entran en juego en dicho proceso vital. De hecho el ámbito neuronal es uno de ellos, por eso la invitación a su estudio y revisión.
\end{abstract}

\section{Palabras clave: FORMACION DOCENTE/ PEDAGOGIA/ EDUCACION/ NEUROCIENCIA/}

Abstract: The following paper seeks to get attention in the incorporation of study of human brain inside of formation for teaching. The capacity of education of each human being has its explanation in the contribution of several scientific disciplines; however sometimes we only focus in psychological and philosophical aspect without take into account the role of biological- human develop. Some situations at once daily scholar has its explanation in the neuronal activity. That is why, the brain-knowledge that teacher could have around its characteristics, potentialities and implications in the different environments of educational action let to have wide sources to make their pedagogical decisions. To think about professional development of the teacher, demands to create and to promote spaces of construction of knowledge about the way in those human beings educate us and elements that enter in game in this vital process, in fact the neuronal scope belongs so one to them it is necessary to assist it.

Palabras clave: EDUCATIONAL PROFESSIONAL DEVELOP/PEDAGOGY/ EDUCATION/ NEUROSCIENCE/

\section{Por qué incorporar el estudio de la neurociencia}

Este artículo surge como producto del análisis de la investigación "Estrategias didácticas en los planes de formación docente para la educación primaria" que se desarrolló en el marco del Proyecto: Apoyo al mejoramiento de la formación inicial de docentes de la educación primaria básica" de la Cooperación Educativa y Cultural Centroamericana. Entre

\footnotetext{
${ }^{1}$ Candidata a Doctora del Programa Latinoamericano de Doctorado en Educación de la Universidad de Costa Rica. Magíster en Evaluación Educativa, Licenciatura en Ciencias de la Educación con énfasis en Administración Educativa y Bachiller en Educación Preescolar, todos los títulos en la Universidad de Costa Rica. En la actualidad labora para el Departamento de Docencia Universitaria de la Escuela de Formación Docente, Investigadora del Instituto de Investigación en Educación, dentro de sus actividades se encuentran el desarrollo del curso Didáctica Universitaria para profesores de la Universidad de Costa Rica.
}

Correo electrónico: suagomez@racsa.co.cr

Artículo recibido: 13 de mayo, 2005

Aprobado: 27 de junio, 2005 
otros, los resultados de este estudio mostraron los ámbitos que se favorecen en la formación del docente de este nivel, a partir de las contribuciones que cada curso aportó según la opinión de los estudiantes. No obstante, no se muestra evidencia acerca del aspecto biológico como parte integrante de los procesos de aprendizaje. El propósito de este artículo es precisamente discutir la necesidad de considerar este ámbito, propio de la integralidad humana y, específicamente, concentrarse en el abordaje del desarrollo neuronal y sus implicaciones para el acto educativo.

La formación de docentes supone plantear la formación en pedagogía y, por ello, este proceso debería fundamentarse en la naturaleza científica de esta última. La pedagogía como ciencia, rescata la necesidad de hacer síntesis de los aportes que otras ciencias brindan al desarrollo de lo educativo. La educación como acción humana no puede comprenderse, sin asumir su carácter complejo de ahí que su acceso y estudio requieren una lectura interdisciplinaria que preste atención a todos aquellos campos científicos que, con su desarrollo teórico y tecnológico, realizan aportes significativos para su comprensión.

Si partimos del hecho de que la pedagogía busca intencionalmente la transformación de las estructuras de conciencia del ser humano (Gállego-Badillo, 1992), es necesario entender que el proceso de construcción del conocimiento es una amalgama de lo mental (que involucra lo biológico) y lo cultural. En el ejercicio pedagógico, los docentes desarrollan un entramado de acciones, que en conjunto con los estudiantes, producen la transformación de saberes, valores y habilidades. Estos procesos permiten vivencias integradas en lo mental y lo cultural.

En el 2002, se desarrolló un proceso investigativo que tuvo como propósito el análisis de las estrategias didácticas utilizadas en los cursos del área pedagógica de la currícula preescrita de los planes de formación de docentes para educación primaria en las universidades estatales costarricenses (Hernández, Montenegro, Francis y Gonzaga, 2002). Este análisis permitió indagar el proceso didáctico de la formación docente, desde las propuestas programáticas de los cursos del área pedagógica de cada plan, a partir de los resultados, se entrevistaron cuarenta y siete docentes responsables de los cursos y ciento sesenta y seis estudiantes de cuarto y quinto años de las carreras de Bachillerato en Educación Primaria de las universidades públicas costarricenses. Este estudio consideró las sedes regionales de dos de las universidades.

De acuerdo con los resultados de esta investigación, los cursos del área pedagógica de los planes de estudio de formación docente de las universidades estatales costarricenses evidencian una atención fundamental en los planos psicológico, filosófico, didáctico y 
curricular. Existe un foco importante en la revisión de los procesos de desarrollo que un infante vive para llegar a aprender, desde su perspectiva psicológica, sociológica, curricular y didáctica. Aunque el desarrollo neurológico del ser humano tiene íntima relación con su proceso de aprendizaje, este no se hace evidente, incluso los docentes en formación, que participaron de este estudio, no lo consideran como contribución de ninguno de los cursos del área pedagógica, lo cual se observa en la Figura 1. No obstante, la investigación en el campo de la neurociencia lo considera parte fundamental del proceso de aprendizaje, por tanto, los docentes requieren discutir y reconocer sus implicaciones en el acto pedagógico. El cerebro es uno de los principales detonadores de la actividad mental que permite el conocer... el educarse.

\section{Reconociendo el aporte de la neurociencia para el trabajo en el aula.}

El carácter biológico y cultural que conlleva el ser humano, confiere al estudio de su capacidad de educabilidad, un sentido complejo y de gran amplitud. Si bien la psicología, la sociología y la antropología han sido prodigiosas en cuanto al entendimiento y operacionalización de la enseñanza, el cerebro y sus funciones parecen haber sido el gran ausente. Morín (1999, p. 20) señala que:

... El hombre sólo se completa como ser plenamente humano por y en la cultura. No hay cultura sin cerebro humano (aparato biológico dotado de habilidades para actuar, percibir, saber, aprender), y no hay mente (mind), es decir capacidad de conciencia y pensamiento sin cultura. 
Figura 1: Categorías de contribución de los cursos del área pedagógica de los planes de formación docente en educación primaria de las universidades públicas costarricenses, según los estudiantes.

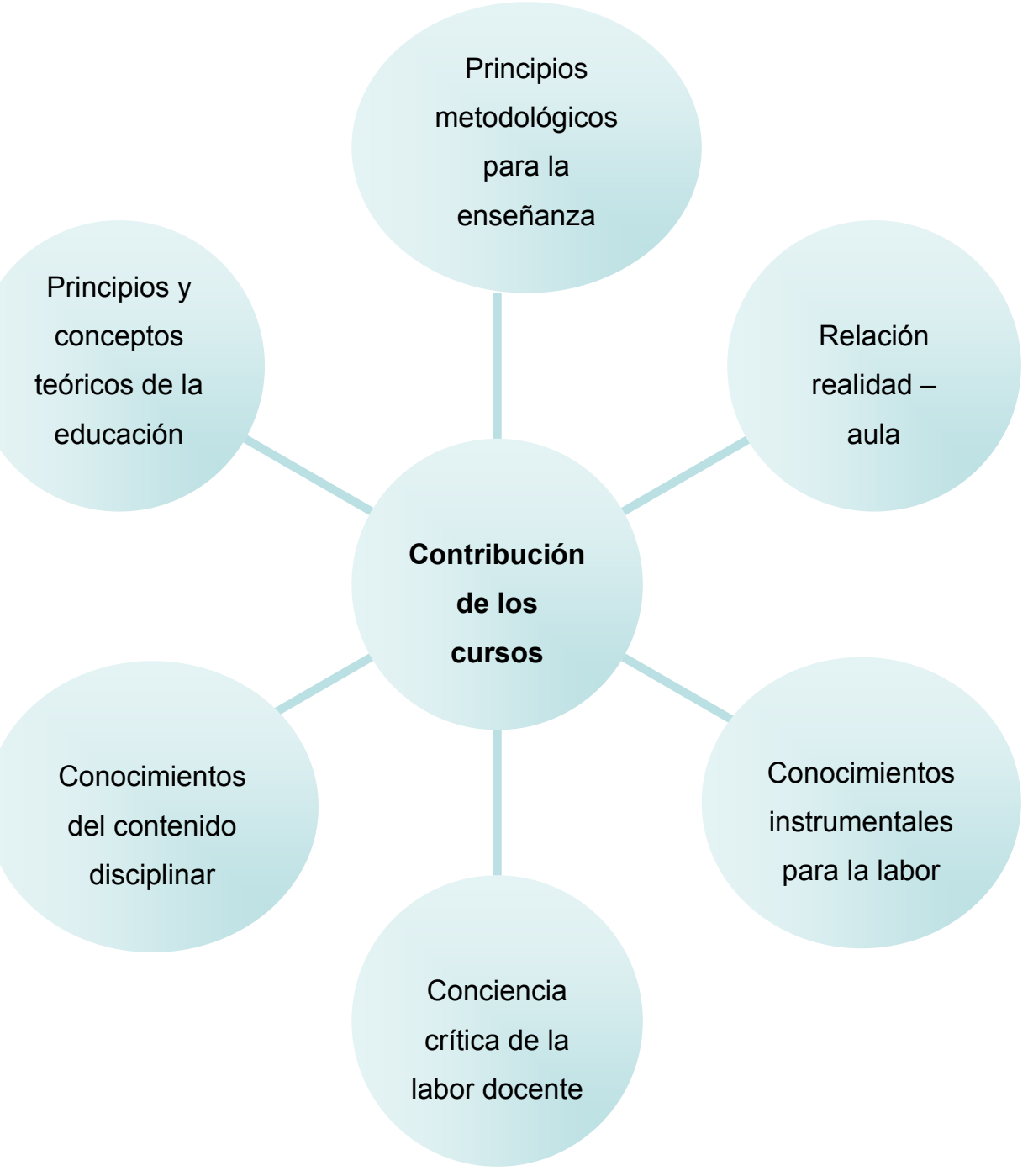

Fuente: Informe Investigación Estrategias didácticas en los planes de formación docente para la educación primaria, 2003, CECC

Si este bucle cultura-cerebro, como lo denomina Morín (1999), interrogara nuestros sistemas de formación docente para la educación inicial, probablemente el silencio daría constancia de que, el cerebro y su estudio para comprender las capacidades del ser humano en la construcción del conocimiento, no han sido invitados al proceso de formación pedagógica de nuestros docentes. Parece que nuestra atención ha sido dirigida al plano 
filosófico, sociológico y psicológico de lo mental y no a su plano biológico, el cual indiscutiblemente no puede separarse de lo mental.

La capacidad de educabilidad que nos caracteriza como seres humanos reside en la capacidad compleja del cerebro que tenemos. Tal y como lo afirman García y García (2001, p. 197) "aún cuando el actor del comportamiento es la totalidad del sujeto y ningún cerebro aislado del resto del cuerpo puede tener actividad mental, es esta víscera el órgano fundamental en la elaboración y gobierno del comportamiento humano".

Es necesario también indicar que la mente o "estado mental" pertenece a una clase de estados funcionales del cerebro en los que se generan imágenes cognitivas sensomotoras que incluyen la autoconciencia. Con ello llamamos la atención de que el cerebro tiene otros estados funcionales que utilizan el mismo espacio de la masa cerebral, pero que, no generan conciencia. Por ejemplo, cuando se esta anestesiado la conciencia desaparece, pero el cerebro sigue funcionando. La mente representa las actividades cerebrales que son isomorfas con el estado del mundo que nos rodea mientras lo observamos, lo reconstruimos, lo transformamos y lo modificamos, o sea, las actividades que coinciden con la representación del mundo externo, por eso la mente es codimensional con el cerebro (Llinás, 2003).

Esta forma de concebir la mente reconoce la necesidad del estudio del cerebro en los procesos de aprendizaje del ser humano, actividad que es consustancial al proceso educativo.

¿Por qué considerar el estudio de las funciones cerebrales en los procesos educativos? La respuesta parece obvia, si se considera que la labor pedagógica es inminentemente humana y esto significa que se debería considerar su integralidad, pero aún así, podemos esbozar dos argumentos:

- En un acto educativo, donde existen diversos procesos comunicativos, se reconoce que toda percepción implica una elaboración, una interpretación, una representación, una construcción del sujeto. Esto nos sitúa frente a un número de construcciones igual al número de personas que estén en dicho acto educativo. Esto justifica que el acto pedagógico se fundamente en la actividad del aprendiz, además nos indica que la actividad mental y la cultural interactúan y se interestructuran para reconstruir y cualificar al mismo ser humano. Flórez (1995) indica que no se puede separar ninguna de estas dimensiones (la mental y neuronal), ni reducir una en detrimento de la otra, se trata más bien de una unidad dual en constante interacción entre mente y cerebro. 
- Tal y como lo indican Rivière y Nuñez (1996), existe una excesiva polarización inconsciente entre lo cognitivo y lo social del desarrollo que, en ocasiones, no atiende los procesos biológicos que facilitan la construcción del conocimiento, mediante los procesos de aprendizaje. Con esto se corre el riesgo de caer en una visión muy limitada de la inteligencia humana, puesto que ésta no puede comprenderse despojada de una matriz social, ni la conducta social e interpersonal pueden entenderse sin explicar a fondo sus requisitos cognitivos.

Ambos argumentos requieren una mirada cuidadosa, sobre todo, si vemos sus implicaciones en la construcción cultural. Si atendemos lo que Gállego- Badillo nos expresa, al plantear que el pedagogo busca la transformación intelectual de la estructura de conciencia, la estructura de saberes, es necesario reconocer cómo esta dimensión neurológica interviene en dicha transformación. Más de la mitad de las investigaciones actuales en la neurociencia, son sobre el aprendizaje y la memoria, lo cual sugiere un gran potencial para apoyar a los educadores en su labor (King-Friedrichs, 2001). Para Wolfe (2001), la información que la neurociencia aporta, provee información básica para la toma de decisiones en estrategias de trabajo en el aula y considera que es una de las dimensiones fundamentales para alcanzar un proceso pedagógico pertinente y significativo.

Asimismo, el estudio del cerebro podría brindar a los docentes, herramientas conceptuales que fundamenten las decisiones que orientan las acciones metodológicas, tanto en el ambiente pedagógico como institucional. La necesidad de vincular en el acto pedagógico, la cultura con el cerebro, se encuentra basada en las siguientes premisas, que apoyan la comprensión de las implicaciones educativas del estudio del cerebro para la labor docente:

\subsection{La fisiología del cerebro y su relación con el proceso de construcción del aprendizaje}

El cerebro como órgano presenta una maravillosa estructura que le permite ser una de las fuentes principales de todo comportamiento humano. Desde las conscientes como el pensamiento, la cognición, memoria hasta las inconscientes como la respiración y la secreción hormonal, todas ellas son actividades que encuentran su fuente de funcionamiento en el cerebro.

Para comprender este órgano, es necesario conocer algunos elementos de su estructura y funcionamiento. El cerebro junto con la médula espinal forman parte de lo que 
se denomina el Sistema Nervioso Central (SNC). Este sistema está conformado por dos tipos de células: las neuronas y las gliales. Las neuronas tienen dos importantes diferencias con respecto a las otras células corporales. Primero no pueden regenerase como lo hacen, por ejemplo, las células epiteliales (las de la piel). Aunque recientemente se creía que los humanos nacíamos con todas las neuronas que íbamos a tener, estudios han demostrado que el cerebro adulto genera nuevas neuronas, aunque se desconoce los procesos de cómo ocurre y cómo funcionan estas nuevas células (Wolfe, 2001).

Segundo las neuronas tienen capacidad de transmitir información y conformarse en redes mediante señales químicas y eléctricas, lo anterior exige una estructura morfológica distinta a las demás células corporales. Su membrana es la que posibilita la transmisión entre las distintas neuronas, su configuración incluye un cuerpo celular (soma) compuesto por el núcleo, múltiples ramificaciones llamadas dentritas y un único axón. La función de las dentritas es recibir información de otras células y la del axón es enviar información a otras células. Ese proceso de compartir información, se denomina sinapsis y es donde se producen señales bioquímicas denominadas neurotransmisoras (García y García, 2001). Los neurotransmisores junto con los receptores controlan la comunicación entre las redes neuronales.

Las células gliales, son denominadas células no excitables que además de dar sostén y mantenimiento al SNC también tienen como función la retirada y "reciclamiento" de moléculas en la actividad sináptica y en la aportación de materiales para la síntesis de neurotransmisores (Wolfe, 2001; García y García, 2001)

El proceso de aprendizaje permite al sujeto su adaptación al entorno cultural. Este proceso requiere de cambios en el sistema nervioso, de ahí la necesidad de la plasticidad del cerebro para poder tener capacidad de aprendizaje y de adaptarse a nuevas situaciones. La capacidad de aprender también está mediada por la memoria que posibilita el aprendizaje por la experiencia. Aunque se ha querido homologar este proceso con el procesamiento de información de las computadoras, nuevas aportaciones de la neurociencia, la psicología cognitiva y la psicología evolutiva han demostrado que no se puede reducir la capacidad cerebral a la metáfora de la computación.

Los procesos de memoria que le permiten al ser humano codificar, almacenar, retraer e integrar nueva información con la ya almacenada, parecen operar en tres categorías interrelacionadas denominadas memoria sensorial, memoria funcional y memoria de largo plazo. El papel de la memoria sensorial es llevar la información nueva al cerebro a través de receptores sensoriales y mantenerla ahí por una fracción de segundos hasta que una 
decisión es tomada sobre qué hacer con esta. La memoria funcional nos permite integrar la información actual percibida con el conocimiento almacenado y concientemente manipular la información, posiblemente para asegurar si lo almacena en la memoria de largo plazo. (Wolfe, 2001)

Por último la memoria de largo plazo tiene una capacidad desconocida hasta ahora, pero es considerada extremadamente grande, algunos estiman que contiene más de un millón de billones de conexiones que le permiten una permanencia a la información. Esta categoría de memoria recibe información elaborada y organizada por la memoria funcional, y a través de dos tipos de memoria, la declarativa que permite almacenar y evocar información que podemos declarar, o sea, decir o escribir y la de procedimiento que facilita el almacenar procesos para acciones de rutina, juntas permiten al sujeto recuperar la información o las habilidades. Sprenger (1999) señala que la memoria de largo plazo incluye además la memoria episódica que se asocia con el recuerdo del contexto, de lo espacial, la ubicación y la memoria automática, identificada recientemente y que frecuentemente está referida a la memoria de respuesta condicionada.

Estos procesos de memoria que acompañan el proceso de aprendizaje se desarrollan gracias a las redes sinápticas que el cerebro produce. Se ha estimado que el $70 \%$ de la cantidad de neuronas (aproximadamente $14 \times 10^{11}$ neuronas) se encuentran en la corteza cerebral. Esto representaría alrededor de $10 \times 10^{13}$ sinapsis en el cerebro humano. También se debe considerar que entre las neuronas existe una organización diferenciada y una diversidad morfológica que permite la especialización de las acciones neuronales (García y García, 2001).

\subsection{El cerebro integra diversos modos de representación de la realidad}

Los pensamientos, emociones, imaginación y predisposiciones operan simultáneamente; estos interactúan con otros modos de adquisición y transformación de la información y con el aumento del conocimiento general, tanto social como cultural. Para la docencia, lo anterior implica el uso de estrategias de didácticas que les permita a los estudiantes orquestar experiencias de aprendizaje donde todos los aspectos de la operación del cerebro se consideren.

En los procesos pedagógicos la atención, por ejemplo, se considera fundamental. En este caso, las emociones y la novedad se reconocen como dos elementos que permiten el logro y mantenimiento de niveles de atención. Tanto las emociones como la novedad, 
interactúan en el espacio neuronal y se ponen de manifiesto fundamentalmente, mediante expresiones comunicativas del lenguaje.

Varios elementos afectan la condición de la atención, entre ellos la dieta, las emociones mismas y las hormonas. Sprenger (1999) señala que la proteína permite al cerebro altos niveles de atención, puesto que ésta le suministra los aminoácidos para producir los neurotransmisores de la atención: dopamina y norepinefrine. Una alimentación adecuada, provista de una cantidad apropiada de proteína, puede significar para el estudiante niveles significativos de atención.

¿Cuánta atención pone un docente a la dieta de sus estudiantes? Por ejemplo, posterior a los recesos, durante los cuales es común el consumo de alimentos, ¿qué sucede con los niveles de atención en el aula? En Costa Rica las instituciones educativas públicas tienen servicios de comedor escolar, los cuales son subvencionados por el Estado y tienen alimentos indicados. La dieta que estos servicios brinden a los niños y niñas tienen consecuencias en el nivel neuronal y, por lo tanto, en los niveles de atención de los niños. Aquí la actividad cerebral se ve afectada, ya no sólo por las decisiones en el aula, sino que se amplia a lo institucional.

Otra faceta del cerebro es que la mayoría de los neurotransmisores están disponibles en mayor cantidad durante la mañana, no así en la tarde. La actividad cerebral tendrá mejores resultados durante este período del día. Esto ofrece importantes implicaciones sobre la naturaleza de situaciones de aprendizaje que son promovidas por el docente, según el horario de trabajo. Las posibilidades de éxito de aquellas estrategias didácticas que requieran mayor atención de parte de los estudiantes, necesariamente deberían tomar en cuenta esta característica del accionar del cerebro. Esta situación es no sólo significativa en el nivel de educación primaria, sino que además llama la atención en los espacios educativos que promueven horarios en la tarde y, sobre todo, en la noche.

\subsection{El aprendizaje compromete la fisiología humana.}

El aprendizaje es tan natural, como lo es la respiración, sin embargo, su desarrollo puede ser perturbado por la nutrición, el estrés y la amenaza entre otros. Reconsideremos de nuevo la nutrición; como ya se indicó, la proteína tiene efectos favorables en la atención, en igual medida los carbohidratos traen consecuencias no tan bondadosas. Por otro lado, Wurtman (citado por Sprenger, 1999) encontró que el $80 \%$ del cerebro consiste en agua, los fluidos son necesarios para mantener fuertes las conexiones entre las neuronas. De esta manera, se resalta la necesaria y adecuada hidratación durante los períodos de clase, 
cuestión que tendrá que tomarse en cuenta cuando se propongan los límites de comportamiento dentro del aula.

Tanto el estrés como la amenaza pueden inhibir los procesos de aprendizaje. Según Jensen (1998) cuando una persona es amenazada, el hipotálamo y las glándulas suprarrenales se asocian para liberar adrenalina, cortisol y vasopresina. Tal situación genera reacciones distintas, pero no produce aprendizaje, el impacto de los químicos liberados se traduce a corto plazo, en daños en la memoria espacial- episódica y debilita la habilidad para establecer prioridades. Igualmente, existe coincidencia en los estudios del cerebro, en indicar que los premios y castigos disminuyen, en igual medida, la motivación. Una muestra de lo anterior se puede hallar en las experiencias desarrolladas por el JUNJI en Chile sobre la Pedagogía del Buen Humor (JUNJI, 2000).

Los docentes que comprenden estos aspectos, considerarían de forma distinta, la formulación y ejecución de límites disciplinarios y normas de interrelación dentro y fuera del aula. Un clima favorable para el aprendizaje requiere aumentar el nivel de endorfina, dopamina, norepinefrine y los niveles de serotonina en sus cerebros por medio de experiencias educativas más placenteras y gratificantes (Wolfe, 2001). El conocimiento de la necesidad y el manejo del estrés, nutrición, ejercicio y relajamiento podrían ser promovidos en el proceso de formación docente, no como elementos periféricos sino como aspectos fundamentales para el logro del aprendizaje.

\subsection{La búsqueda de lo significativo es innata}

Los ambientes de aprendizaje requieren estabilidad y familiaridad. El aprendizaje permite la generación de redes conceptuales de conocimiento que la memoria almacena como estructuras proposicionales. La determinación de qué es significativo y qué no, está reflejado no sólo en el proceso perceptivo inicial, sino también en el procesamiento consciente de la información. Los mecanismos de almacenamiento se pueden describir como redes de asociaciones (Wolfe, 2001).

Cuando se incorporan nuevos aprendizajes, estos se vinculan con experiencias pasadas. Dicha conexión puede ser significativa y va a depender del grado de vinculación y familiaridad con las redes conceptuales almacenadas. La adquisición de nueva información produce nuevas conexiones sinápticas; cada cuerpo celular o neurona tiene ramificaciones denominadas dentritas y una simple proyección denominada axón. Esta última se conecta con las dentritas de otra neurona formando las sinapsis. Estas conexiones se forman y se 
fortalecen cuando las experiencias son familiares e incorporan elementos novedosos y coherentes para el sujeto que aprende.

El aprendizaje es uno de los mayores vehículos para la adaptación comportamental y es una poderosa fuerza para el progreso social (Kandell \& Hawkins, 1992). Las estrategias que permitan el contacto con la realidad y la reiteración favorecen el paso de información de la memoria de corto plazo a la memoria de largo plazo; esto hace que tenga sentido y significado (King-Friedrichs, 2001). Por ello, aquellas estrategias didácticas que promuevan la vinculación con la realidad y la pre - exposición a una información novedosa, han demostrado una vía para familiarizar al estudiante con los contenidos disciplinares en estudio.

Los recursos conceptuales y tecnológicos didácticos requieren satisfacer la curiosidad cerebral y el apetito por el descubrimiento y desafío. Las entregas didácticas necesitan ser excitantes, significativas y deberían proporcionar opciones divergentes dentro de la realidad que vive el sujeto aprendiz.

\subsection{La búsqueda de los significados se da por medio de modelos}

El cerebro es tanto artista como científico (Collins, 2001), está diseñado para percibir y generar patrones para representar las realidades que construye, asimismo, se resiste a tener patrones que no tengan sentido o bien que sean impuestos. La construcción de estos patrones de representación, requiere la asociación con la información que ya está almacenada y procesada. Por eso cuando no hay posibilidad de esta asociación los patrones de representación no llegan a tener sentido y se convierten en partes aisladas de información que no se relacionan con lo significativo para un estudiante.

Las conexiones sinápticas son con frecuencia temporales, el espacio neuronal es la propiedad más costosa del cuerpo humano, así que el cerebro construye sinapsis sólo cuando son necesarias. Por tanto, para asegurar que el cerebro mantiene las conexiones sinápticas necesarias se requieren elaboraciones que fortalezcan la asociación entre el primer contacto con la información y las representaciones que forman parte de los patrones que ya tiene el cerebro, para así mantener su significatividad. El uso en el aula de estrategias didácticas que promuevan las clasificaciones, análisis, pruebas y profundizaciones permiten brindar retroalimentación sobre qué tan bien se está dando esta asociación comprensión.

La promoción de estrategias didácticas para que el estudiante desarrolle procesos metacognitivos, ofrece una oportunidad para probar patrones de pensamiento. Los cerebros 
de los estudiantes desarrollan mejores patrones de pensamiento cuando estos han tenido espacios para probar de forma minuciosa modelos para el aprendizaje que ya han sido probados en la realidad (Jensen, 1998). El caso del aprendizaje de la lectura y la escritura ilustran el modelaje que utiliza el cerebro en sus construcciones. De acuerdo con D'Arcangelo (2003) los niños y adultos para leer utilizan típicamente tres sistemas cerebrales ubicados en el lado izquierdo, hemisferio que tradicionalmente se dedica al lenguaje. Al aprender a leer, primero se hacen conexiones para llegar a construir un modelo neurológico de las palabras, el cual se construye clarificando las representaciones de cada palabra.

\subsection{Las emociones son críticas en la elaboración de patrones de aprendizaje.}

No podemos perder de vista que los seres humanos somos unidades bio-sociales; por eso las emociones son cruciales en los procesos de aprendizaje. Lo que aprendemos está influenciado y organizado por emociones y estructuras mentales basadas en expectativas, inclinaciones y prejuicios personales, grados de autoestima y la necesidad de interactuar socialmente. La emoción y la cognición no pueden separarse. En palabras de Maturana (1997) la existencia humana se realiza en el lenguaje y lo racional desde lo emocional, porque los actos humanos se fundamentan en los argumentos que construimos al concatenar las acciones que son significativas para nosotros, son espacios de acciones que son especificados desde una emoción, así mismo está fundado el razonar.

Cuando los estudiantes están emocionalmente comprometidos con el aprendizaje, ciertos neurotransmisores en el cerebro envían señales al hipocampo, estructura vital del cerebro que incluye la memoria, para estampar estos eventos con gran intensidad. (Cahill citado por King-Friedrichs, 2001) Un elemento asociado con la evaluación formativa es la realimentación en el proceso, al darse una retroacción favorable para un estudiante, este eleva los niveles de serotonina. La serotonina es uno de los neurotransmisores mejor conocidos; actúa como un intensificador del humor, pues calma, más que desequilibrar el cerebro, su notoriedad reside en el control y disminución de la depresión y el estrés. La serotonina se relaciona con la memoria, el sueño, el control del apetito y la regulación de la temperatura del cuerpo, por ello, posibilita estados de atención y relajación necesarios en procesos de aprendizaje. En consecuencia las acciones de amenaza hacia la persona, provocan bajos niveles de este neurotransmisor. La carencia de este neurotransmisor ha sido vinculado especialmente con comportamientos agresivos tales como combate, provocación de incendios y hasta suicidio. 
Otra faceta de las emociones demuestra que éstas excitan el sistema químico cerebral $y$, en conjunto con la adrenalina liberada, actúan como un fijador en la memoria. El clima afectivo y emocional de la institución educativa y de la clase es altamente significativo; utilizar estrategias efectivas de comunicación y permitir a los estudiantes y docentes la reflexión sobre los procesos metacognitivos que supongan experiencias de éxito, son detonadores de una acción cerebral que enriquece los procesos de aprendizaje de las personas.

\subsection{El cerebro integra partes y todos simultáneamente}

Existe evidencia que plantea diferencias significativas entre las acciones del hemisferio izquierdo y el derecho. En una persona saludable, los dos hemisferios son indisolublemente interactivos, aún cuando una persona está repartida en palabras, matemáticas, música o arte. Las personas tienen enormes dificultades de aprendizaje cuando ya sea las partes o el todo, son pasados por alto. Cuando hablamos del proceso de lenguaje, por ejemplo, este se da por medio de tres grupos interactivos de estructuras. La primera es una gran colección de sistemas neuronales en ambos hemisferios que representan las interacciones no verbales entre el cuerpo y su ambiente, mediados por una variedad de sistemas sensoromotrices. La segunda es un pequeño número de sistemas neuronales ubicados en el hemisferio izquierdo que representa fonemas, sus combinaciones y reglas sintácticas de combinación de palabras. El tercer grupo de estructuras están también localizadas en el hemisferio izquierdo, mediadas por las dos anteriores, este grupo puede llevar un concepto y estimular la producción de palabras o bien, recibir palabras y provocar que el cerebro evoque sus correspondientes conceptos (Damasio \& Damasio, 1992). D’Arcangelo (2003) también responsabiliza a este grupo de estructuras del proceso que permite el aprendizaje de la lectura y escritura

Esta premisa tiene importantes implicaciones en el abordaje de estrategias didácticas tan particulares, como los mapas conceptuales o las uves heurísticas propias del paradigma cognitivo y promovidas por Novak (1998) o bien los esquemas mentales, que a partir de la combinación de colores, palabras, imágenes intentan desarrollar actividad en ambos hemisferios. Si se comprende qué tipo de ambiente favorece estas estructuras en el cerebro, el docente podrá comprender la importancia de ambientes letrados, de ricas comunicaciones, retroalimentaciones que permitan el control y la autorregulación en el aprendizaje, con base en una gran cuota de participación de los estudiantes. La promoción de este tipo de 
ambiente permitiría la construcción de amplias y complejas redes conceptuales con lo cual se procuraría el desarrollo niveles de inteligencia más elevados.

\subsection{El aprendizaje siempre involucra procesos conscientes e inconscientes}

Muchas de las señales que son periféricamente percibidas por el cerebro, ingresan a este, sin el conocimiento del aprendiz e interactúan en niveles de inconciencia. Crack y Koch (1992) indican que las personas que disfrutan de una visión normal, responden con regularidad a las señales visuales sin ser conscientes de ello. En acciones automáticas como las de nadar o conducir se efectúan complejos, pero a la vez, estereotipados movimientos a los que se asocia una escasa, y en ocasiones, alguna conciencia visual. Para llegar a tener una percepción consciente se requiere tiempo.

Podemos evocar nuestras experiencias y recordar lo que hemos vivido, pero no lo que hablamos. Estudios sobre rendimiento académico (Chen \& Stevenson, 1995; Steinback \& Gwizdala, 1995; Contreras, 1994, Moreira, 2001), han demostrado que la actitud hacia la matemática que los estudiantes han construido, afecta significativamente su rendimiento académico en dicha asignatura. No obstante, es más preocupante saber que dicha actitud está siendo cultivada en su mayor parte en el aula, sobre todo con las expresiones que los docentes hacen acerca del nivel de dificultad que puede representar esta disciplina. Tales expresiones se incorporan en las estructuras mentales y se expresan inconscientemente.

Un estudio realizado por Dartmouth College, guiado por Reginald Adams (Collins, 2001), demostró que el cerebro interpreta las expresiones de los rostros de manera diversa según la dirección de las miradas, en este caso cuando una persona mira directamente a otra persona. Según esta investigación la dirección de la mirada influencia nuestro cerebro, ya que responde a emociones de miedo, enojo, expresadas en el rostro de la otra persona. En particular, la dirección de la mirada influye sobre la amígdala, región del cerebro que regula las emociones y dirige el comportamiento emocional.

Las formas de actuación humanas están guiadas en su mayor parte, por la memoria más que por la información sensorial inmediata, y dependen de una constante actualización de la información pertinente. Las posibilidades de respuesta de un estudiante no sólo involucran contenidos, sino que habilidades, valores y creencias alrededor de dicho contenido. La capacidad para formar conceptos abstractos para un estudiante, puede depender de una fundamental aptitud para almacenar representaciones del mundo exterior y para responder a estas representaciones, aunque los objetos no estén presentes (Goldman Rakic, citado por Collins, 2001). 


\subsection{Los tipos de memoria: el sistema de memoria espacial o contextual y un grupo de sistemas para el aprendizaje repetitivo.}

Los seres humanos cuentan con un sistema de memoria espacial que no requiere recitación y permite la memoria instantánea de experiencias. Este sistema siempre está comprometido y es inagotable. También tenemos un grupo de sistemas diseñados para almacenar información que no está vinculada entre sí. Este último sistema depende de la memoria reiterativa y de la acción ensayada, y tiene como función la separación de la información y las habilidades del conocimiento importantes extraídas de la experiencia actual.

Sprenger (1999) señala al respecto, que estudiantes que aprenden información en un salón y son "evaluados" en otro, obtienen calificaciones más bajas que aquellos que son evaluados en el mismo salón. Igualmente, reseña que investigadores han encontrado que los estudiantes tienen más dificultad en resolver problemas de matemática cuando se encuentran en clase de inglés. Los investigadores llaman a esto "información invisible" (Schenger, 1998)

El enfoque pedagógico que ha imperado en el aula escolar enfatiza estrategias que enfatizan más la repetición de datos desvinculados y sin sentido para los estudiantes. Dicha acción no facilita la transferencia del aprendizaje y probablemente interfiere con el desarrollo subsiguiente del entendimiento, inhibiendo de esta manera el funcionamiento efectivo del cerebro.

Nuestro lenguaje es aprendido por medio de múltiples experiencias interactivas que envuelven vocabulario y gramática. Este es moldeado por procesos internos y la interacción social (Vigotski, 2001) El lenguaje es muestra de cómo los aspectos específicos dan significado cuando se encuentran integradas a experiencias ordinarias. La educación, por tanto, puede transformarse, si este tipo de integración se intensifica. La inclusión de demostraciones, proyectos, viajes de campo, imaginario visual de ciertas experiencias y mejores desempeños, historias, metáforas, dramas procuran la interacción de diferentes áreas de contenido e intensifican la asociación de las experiencias con las construcciones cerebrales y las actividades de la vida real.

\subsection{El aprendizaje se intensifica por el desafío e inhibiendo la amenaza.}

El cerebro baja sus niveles de acción cuando percibe amenaza, por el contrario, este se activa en forma óptima cuando es apropiadamente desafiado. La principal señal del descenso de la actividad es un sentimiento de impotencia, el aprendiz llega a ser menos 
flexible y se revierte a lo automático y, frecuentemente, a las más primitivas rutinas de comportamiento. La exposición a constantes amenazas o traumas tempranos, frecuentemente alteran el comportamiento neuronal, creando niveles extremos de serotonina y noradrenalina.

La propuesta desarrollada por la Junta Nacional de Jardines Infantiles (JUNJI, 2000) de Chile, denominada la Pedagogía del Buen Humor, plantea importantes consideraciones alrededor de esta premisa. De acuerdo con la conceptualización teórica del humor (como situación antónima de amenaza), la imagen provocada por situaciones humorísticas, llega a través de la corteza cerebral hasta el tálamo y el hipocampo, y hacia los centros emocionales de la amígdala; de allí la información pasa a estimular el cuerpo calloso y el cortex prefrontal, donde tienen lugar los procesos cognitivos. Cuando una persona es consciente de que algo es gracioso, en su actividad mental ha precedido un proceso de confrontación de nueva información con la base que ya existe, se produce entonces, una incongruencia con lo esperado, o con la información que habitualmente tenemos. Se produce una acción cerebral que predispone para mejores aprendizajes. La inclusión de esta categoría permitió, al equipo del JUNJI, identificar importantes beneficios en el aprendizaje fundamental relacionados con autoestima, confianza, seguridad, creación, expresión, lenguaje y convivencia.

\subsection{Cada cerebro es único}

Mientras todos los seres humanos tenemos los mismos sistemas fisiológicos, estos están integrados en forma diferente en cada cerebro. Debido a que el aprendizaje cambia la estructura cerebral, cuanto más aprendamos, más complejo llega a ser nuestro cerebro.

Al respecto, Jensen indica que los mejores aprendizajes incluyen curiosidad, anticipación y desafío. Cada estado se encuentra definido por una única formulación química neuronal que incluye los neurotransmisores como la dopamina y hormonas como la adrenalina. Por su parte, Sprenger señala que las diversas estimulaciones cerebrales pueden producir diferentes resultados $\mathrm{y}$, por ello, se plantean distinciones entre los aprendices; por ejemplo los kinestésicos necesitan mayor movimiento; los auditivos requieren conversar sobre el material.

Esto convierte a la enseñanza en una tarea multifacética que permite a los estudiantes expresarse en forma diferente: visual, táctil, emocional, entre otros. Aquí no es distinguir "tipos de inteligencia", sino más bien, el reconocimiento de que los estilos de aprendizaje tienen su base también en la configuración neuronal de cada persona. 


\section{Consideraciones finales}

Las exigencias para la docencia aumentan día con día; ahora se espera que en el espacio educativo, los profesores sean capaces de formar personas con pensamiento crítico, con una expresión clara de sí mimos, capaces de resolver problemas complicados y llegan a ser aprendices para toda su vida. Lo anterior implica sintetizar los aportes que detallan científicamente los aspectos humanos que favorecen el aprendizaje, como la acción con la cual el ser humano conoce.

La síntesis de los aportes de las ciencias humanas, no requiere la creación de nuevos cursos dentro de los planes de formación docente, sino de la incorporación del espacio biológico en la comprensión de la formación humana. Pareciera que esta esfera ha venido siendo disminuida por una atención significativa a la parte afectiva y cognitiva, que no obstante como ya lo vimos, también tienen como una de las fuentes de origen, la acción cerebral.

Este llamado de atención más que proponer la "incrustación" de nuevos cursos referidos a esta esfera, intenta proponer un conocimiento integral y fundamentado de la capacidad de educabilidad del ser humano por parte de los formadores de docentes y así abrir las puertas a estos últimos para su lectura y abordaje pedagógico. No es gratuito que los docentes de educación especial, en su afán de comprender a los estudiantes con necesidades educativas especiales, recurran tanto al plano biológico, como al cultural.

Volver los ojos a nuestro funcionamiento bio-físico y químico, no nos separa de la parte mental- emocional, social- cultural, pues somos seres complejos e integrales. El acto pedagógico no puede obviar esta integralidad. Un docente requiere no sólo el conocimiento de la didáctica, del contexto y de la disciplina, sino la comprensión epistemológica y, sobre ésta, el entendimiento de los procesos biológicos por los cuales el individuo transita para construir los conocimientos. La búsqueda de la transformación de las conciencias intelectuales de los individuos, sin comprender la naturaleza bio-química, que también media durante los procesos de aprendizaje, podría resultar en una toma de decisiones pedagógicas no pertinentes, de acuerdo con la intención educativa que se busca.

La toma de tales decisiones requiere la comprensión del objeto teórico disciplinar, de las estrategias didácticas y del entramado psico - biológico - social que los estudiantes presentan. Integrar los avances de la investigación neurológica pretende enriquecer la comprensión que el docente tiene sobre la búsqueda de las transformaciones intelectuales en los seres humanos. En definitiva revela grandes pistas para orientar la mediación docente con la base de una comprensión de lo que los seres humanos representamos: seres bio- 
mentales en constante interacción y fundamenta muchos discursos que, hasta la fecha, parecen más artificios que propuestas pedagógicas.

\section{Referencias}

Damasio, A. R. \& Damasio, H. (1992). Brain and Language. Scientific American. Setiembre 63-71.

D’Arcangelo, M. (2003). On the Mind of a Child. Educational Leadership, 60 (7), 6-11

Chen, C. \& Stevenson, H. (1995). Motivation and Mathematics Achievement: A Compartative Study of Asian- American, Caucasian-American, and East Asian High School Students. Child Development, 66, 1215-1234

Collins R. (2001). Best Practices in Teaching and Learning What does the research say? Recuperado el 23 de marzo, 2003 de www.curriculum.edu.au/scis/connect/cnetw02/cnet14in.htm

Contreras, I. (1995). El quehacer diario del profesor de matemática en la educación secundaria: algunos resultados de su estudio. Revista Educación, 19(1), 61-71.

Flórez, O. R. (1995). Hacia una pedagogía del conocimiento. Colombia: McGraw Hill.

Gallego-Badillo, R. (1998). Saber Pedagógico Una visión alternativa. Colombia: Editorial Magisterio.

García, C. J. y García, D. A. (2001). Teoría de Educación II: Procesos primarios de formación del pensamiento y la acción. España: Ediciones Universidad Salamanca.

Hernández, A., Montenegro, M., Francis, S., Gonzaga, W. (2002). Estrategias didácticas en los planes de formación docente para la educación primaria Revista Centroamericana de Educación, 1(2) 17-33.

Hernández, A., Montenegro, M., Francis, S., Gonzaga, W. (2003). Informe Investigación Estrategias didácticas en los planes de formación docente para la educación primaria. San José: CECC.

Jensen, E. (1998). How Julie's brain learns. The best of Educational Leadership 1998, 31-35.

JUNJI. (2000). Pedagogía del buen humor en Educación Parvularia. Chile: JUNJI, Mimeografiado

Kandell, E., y Hawkins, R. (1992). The Biological Basis of Learning and Individuality. Scientific American, 53-60.

Llinás, R. (2003). El cerebro y el mito del yo. Colombia: Grupo Editorial Norma. 
Maturana, H. (1997). La realidad ¿objetiva o construida? I. Fundamentos biológicos de la realidad. España: ANTHROPOS.

Moreira, M., T. (2001). Percepciones sobre la formación docente y su posible articulación con la enseñanza de la matemática: un estudio de casos. Revista Educación, 25(1), 53-66.

Morín, E. (1999). Los siete saberes necesarios para la educación del futuro. Francia: UNESCO.

Novak, J. (1998). Conocimiento y aprendizaje: Los mapas conceptuales como herramientas facilitadoras para escuelas y empresas. España: Alianza Editorial

Rivière, A. \& Nuñez, M. (1996). La mirada mental. Argentina: Aique.

Steinback, M. \& Gwizdala, J. (1995). Gender Differences in Mathematics Attidudes of Secondary Students. School Science and Mathematics, 95(1).

Sprenger, M. (1999). Learning and Memory. The Brain in Action. Virginia, USA: ASCD.

Wolfe, P. (2001). Brain Matters: Translating research into classroom practice. Virginia: USA: ASCD. 